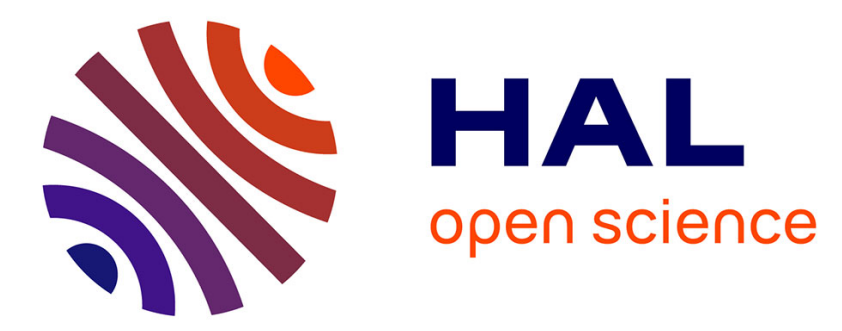

\title{
Periodic solutions of a multi-dof beam system with impact
}

\author{
E.L.B. van de Vorst, D.H. van Campen, A. de Kraker, R.H.B. Fey
}

\section{To cite this version:}

E.L.B. van de Vorst, D.H. van Campen, A. de Kraker, R.H.B. Fey. Periodic solutions of a multi-dof beam system with impact. Journal of Sound and Vibration, 1996, 192 (5), pp.913-925. 10.1006/jsvi.1996.0225 . hal-01635165

\section{HAL Id: hal-01635165 \\ https://hal.science/hal-01635165}

Submitted on 14 Nov 2017

HAL is a multi-disciplinary open access archive for the deposit and dissemination of scientific research documents, whether they are published or not. The documents may come from teaching and research institutions in France or abroad, or from public or private research centers.
L'archive ouverte pluridisciplinaire HAL, est destinée au dépôt et à la diffusion de documents scientifiques de niveau recherche, publiés ou non, émanant des établissements d'enseignement et de recherche français ou étrangers, des laboratoires publics ou privés. 


\title{
PERIODIC SOLUTIONS OF A MULTI-DOF BEAM SYSTEM WITH IMPACT
}

\author{
E. L. B. van de Vorst, D. H. van Campen and A. De Kraker \\ Department of Mechanical Engineering, Eindhoven University of Technology, P.O. Box 513, \\ 5600 MB Eindhoven, The Netherlands \\ AND \\ R. H. B. FEY \\ TNO Centre for Mechanical Engineering, P.O. Box 49, 2600 AA, Delft, The Netherlands
}

\begin{abstract}
The steady state behaviour is analyzed of a periodically driven multi-DOF beam system which has an elastic stop at its middle. The elastic stop is modelled in a continuous way by using the contact law of Hertz. The beam is modelled by using finite elements and subsequently reduced by using a component mode synthesis method. The steady state behaviour of the system reduced to one, two and four DOF's is investigated by calculating periodic solutions at varying excitation frequency. Periodic solutions are calculated by solving two-point boundary value problems by the multiple shooting method in combination with a path-following technique. It is shown that models with more than one DOF are required for a good assessment of the long-term behaviour of the system.
\end{abstract}

\section{INTRODUCTION}

In many practical engineering applications of mechanical systems, impacts at stops occur. It is important to carry out a dynamical analysis of such systems, to identify and subsequently reduce the noise and wear caused by repeated unacceptably large impacts. Examples of systems with impacts are gear rattle, heat exchanger tube wear in nuclear power stations and ships colliding against fenders. Systems with stops are typical examples of systems with strong local non-linearities. Although the non-linearity is local, the overall dynamic response of the system may change drastically. A system with stops cannot be linearized, so it is very difficult to predict the system response without a non-linear analysis.

In general, two different approaches are possible for analysis of the impact in the systems outlined above. One approach is to assume that the impact occurs instantaneously. The analysis is thus divided into two intervals: before and after the impact. The interconnection between the two intervals is made by a momentum balance and a parameter representing the amount of energy dissipation in the impact, called the coefficient of restitution. This approach is used in many analyses of impact oscillators and has led to the identification of bifurcational behaviour which is not found in smooth dynamical systems. Impact rules for such assumed instantaneous reversal of velocity induce discontinuities in the derivatives of maps defined by taking a section through the full phase space. In fact, these discontinuities yield the "new" bifurcational behaviour, called grazing or border-collision bifurcations [1-3]. 
The other approach is to let the collision forces act in a continuous matter. The impact analysis of a system of colliding bodies is hence performed simply by including the collision of the contact forces in the system equations of motion during the contact period. A suitable model for the contact forces during the contact period is the non-linear Hertzian force-displacement law [4]. This model itself does not represent the energy dissipation process. Although the theory of Hertz is based on elasticity, some studies have been performed to extend the theory to include energy dissipation $[5,6]$. In the paper by Foale and Bishop [7] bifurcations occurring in single-DOF impact oscillators in which the impact is modelled by using the Hertzian contact law are compared with "grazing" bifurcations which occur in impact oscillators where the impact is modelled in a discontinuous way.

The main focus of research into impact oscillators in recent years has been into single-degree-of-freedom systems. In this paper we will focus on the influence on the system response of adding more degrees of freedom (DOF's). A beam system is investigated which has an elastic stop at its middle and is periodically excited in the middle. A less complicated version of this beam system, supported in the middle by a massless one-sided linear spring, was investigated earlier by Fey [8], Fey et al. [9] and Van de Vorst et al. [10]. This beam system can be divided into a linear and a non-linear component. The linear component (the beam) is modelled by means of the finite element method and consequently has many more DOF's than the non-linear one. Because the numerical analysis of the resulting non-linear system is very expensive from a computational point of view, in particular for increasing number of DOF's, it is worthwhile to keep the number of DOF's as low as possible. This can be achieved by applying a reduction method to the finite element model of the linear component (the beam). The particular reduction method applied is the component mode synthesis method $[11,8]$ and offers the possibility for a considerable reduction of the DOF's and for a systematic investigation of the influence of adding or deleting DOF's. Moreover, the component mode synthesis method can easily be used for more complex systems. The component mode synthesis method utilized in this paper uses free interface eigenmodes up to a cut-off frequency and residual flexibility modes to approximate the dynamic and static behaviour of the linear component. After reduction of the linear component, the non-linearity is added, resulting in a reduced non-linear system which will be valid for frequencies up to the cut-off frequency used in the reduction procedure. Because generically non-linear systems generate higher frequencies than their excitation frequency, the cut-off frequency has to be chosen much higher than the maximum excitation frequency.

The elastic stop is modelled by using the contact law of Hertz without energy dissipation, as mentioned earlier. Hence, the collision forces act in a continuous way. Periodic solutions of the reduced system are calculated for varying excitation frequency by solving two-point boundary value problems by the multiple shooting mehtod [12] in combination with a path-following method [8]. The response of the system is investigated for models with one, two and four DOF's. Also, different modal damping levels are applied. All calculations presented in this paper were carried out by using a development release of the finite element package DIANA [13].

\section{BEAM SYSTEM WITH AN ELASTIC STOP AT ITS MIDDLE}

The beam system which is analyzed numerically is shown in Figure 1. The beam is supported at both ends by leaf springs. If the displacement $y$ of the middle of the beam positive, the beam hits a spherical elastic contact with radius $r=0.005 \mathrm{~m}$, which is connected rigidly to the outside world. In the middle of the beam also a rotating mass is 


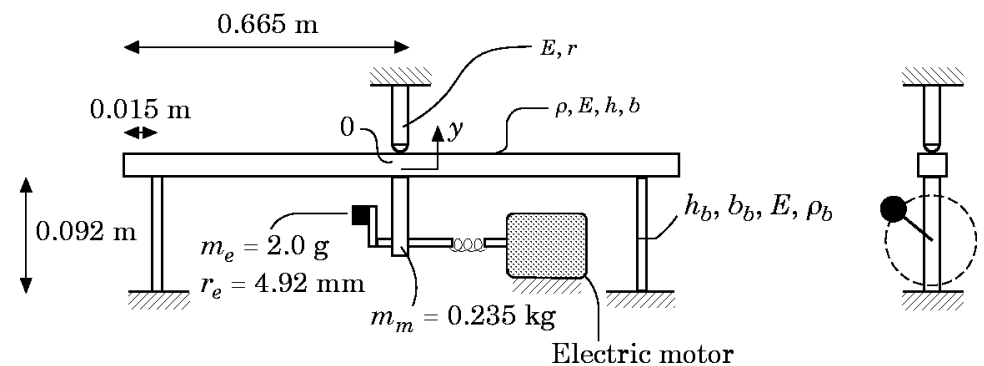

Figure 1. The beam system. $E=2.1 \times 10^{11} \mathrm{~N} / \mathrm{m}^{2}, r=0.005 \mathrm{~m}, h=0.01 \mathrm{~m}, h_{b}=0.001 \mathrm{~m}, b=0.09 \mathrm{~m}, b_{b}=$ $0.075 \mathrm{~m}, \rho=7746 \mathrm{~kg} / \mathrm{m}^{3}, \rho_{b}=7713 \mathrm{~kg} / \mathrm{m}^{3}$.

present generating a periodic force. Energy dissipation is established by a linear damper (not shown in Figure 1) at the middle of the beam (see also section 3).

The beam and leaf springs are modelled by using the finite element method and reduced by using the component mode synthesis method mentioned in section 1 . Because of symmetry, only half the beam is modelled. (The elastic stop and the excitation force are positioned exactly at the middle of the beam. Hence the asymmetric model is linear, which means that symmetry breaking bifurcations cannot occur.) As mentioned in section 1, the contact force $F_{s}$ between the beam and the spherical elastic contact is modelled by using Hertz's law $[4,5]$ : that is,

$$
F_{s}(y)=\left\{\begin{array}{ll}
k_{s} y^{3 / 2}, & y \geqslant 0 \\
0, & y<0
\end{array}\right\} .
$$

In equation (2.1) the parameter $k_{s}$ is taken as $1.034 \times 10^{10} \mathrm{Nm}^{2 / 3}$ for a contact radius of $r=5 \mathrm{~mm}$ and $E=2 \cdot 1 \times 10^{11} \mathrm{~N} / \mathrm{m}^{2}$ for the spherical contact.

\section{SINGLE-DOF MODEL}

After reduction of the finite element model, the equation of motion for the single-DOF model may be obtained as

$$
m \ddot{y}+b \dot{y}+k y+k_{p} y^{3 / 2}=m_{e} r_{e} \omega^{2} \cos (\omega t) .
$$

Here

$$
k_{p}=\left\{\begin{array}{ll}
k_{s}, & y \geqslant 0 \\
0, & y<0
\end{array}\right\}
$$

and $k_{s}=5 \cdot 17 \times 10^{9} \mathrm{Nm}^{2 / 3}, \quad \omega=2 \pi f_{e}, \quad b=2 \xi \sqrt{m k}, \quad \xi=0.0142, \quad m=\left(\boldsymbol{\varphi}_{1}^{\mathrm{T}} \mathbf{M} \boldsymbol{\varphi}_{1}\right) / \varphi_{1 y}^{2}=$ $2.48 \mathrm{~kg}, k=\left(\boldsymbol{\varphi}_{1}^{\mathrm{T}} \mathbf{K} \boldsymbol{\varphi}_{1}\right) / \varphi_{1 y}^{2}=16877 \mathrm{~N} / \mathrm{m}, m_{e}=1.0 \mathrm{~g}$ and $r_{e}=4.92 \mathrm{~mm}$. $\mathbf{M}$ and $\mathbf{K}$ are the mass and the stiffness matrices of the linear part of the system, i.e., the beam without the one-sided spring. $\boldsymbol{\varphi}_{1}$ is the first eigenmode of the linear system with an eigenfrequency of $13 \cdot 1 \mathrm{~Hz} . \varphi_{1 y}$ is the element of $\boldsymbol{\varphi}_{1}$ corresponding to the displacement of the middle of the beam $y$.

Branches of periodic solutions of this system have been calculated for varying excitation frequency $f_{e}$. The maximum absolute displacements $|y|_{\max }$ occurring in these periodic solutions are shown in Figure 2. Besides the harmonic resonance peak near $25 \cdot 3 \mathrm{~Hz}$, the figure also shows subharmonic resonance peaks. In Figure 3 the periodic solution at $f_{e}=25 \cdot 3 \mathrm{~Hz}$ is shown. 


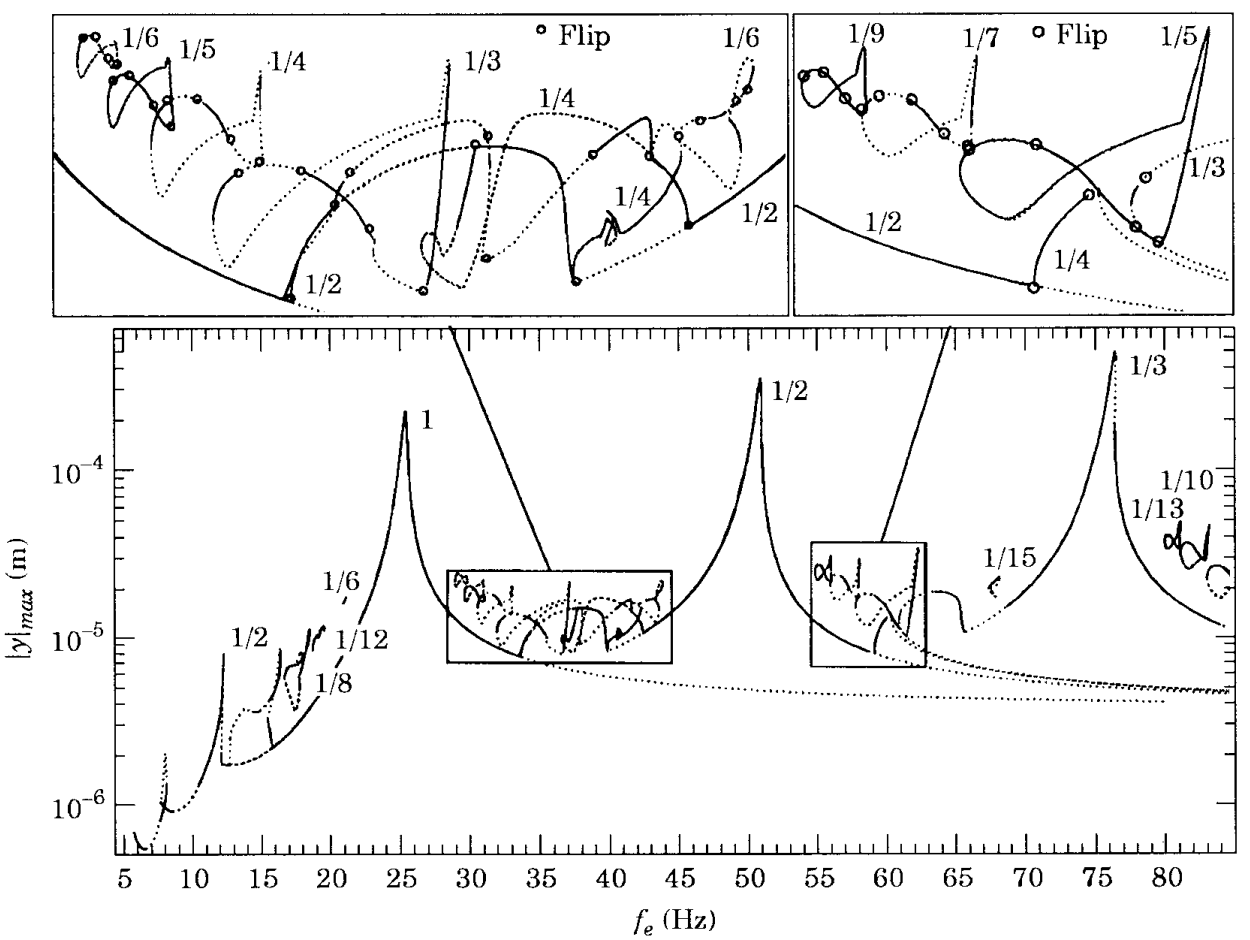

Figure 2. The maximum displacements of the periodic solutions of the 1-DOF model. $\cdots$, Unstable; , stable.

Notice that, compared to the linear system, the first harmonic resonance peak has increased from $13 \cdot 1 \mathrm{~Hz}$ to $25 \cdot 3 \mathrm{~Hz}$. Shaw and Holmes [14] have given the following expression for the bilinear eigenfrequency $f_{b}$ of an undamped bilinear single-DOF system:

$$
f_{b}=\{2 \sqrt{1+\alpha} /(1+\sqrt{1+\alpha})\} f_{1}
$$

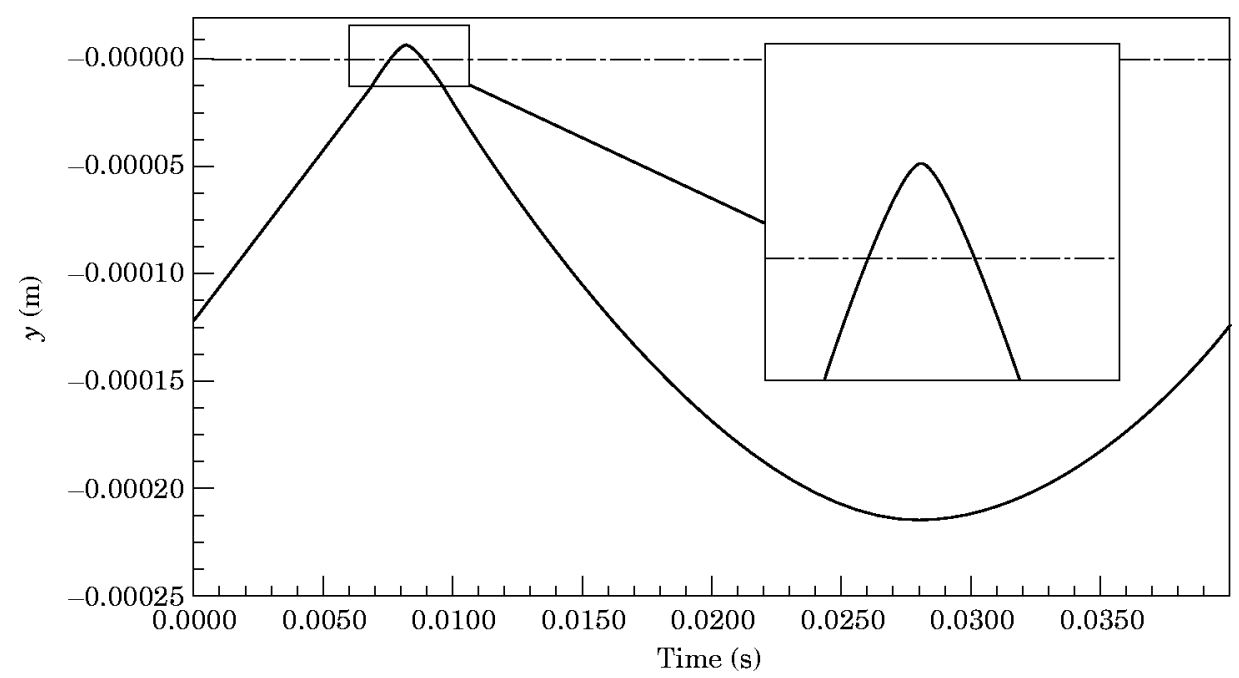

Figure 3. The periodic solution of the 1-DOF model at $f_{e}=25 \cdot 3 \mathrm{~Hz}$. 
Here $\alpha=k_{b} / k$, with $k_{b}$ the stiffness of the bilinear spring. In our system the contact forces are non-linear. If, however, $\alpha$ is calculated from equation (3.2), with $f_{b}=25.3 \mathrm{~Hz}$ and $f_{1}=13 \cdot 1 \mathrm{~Hz}$, this results in $\alpha \approx 790$ and by using this $\alpha$ the contact forces resulting from Hertz's law and from the bilinear system can be compared. In the bilinear case the contact force is $F_{s_{b}}(y)=\alpha k y=790 \times 16877 y=1.33 \times 10^{7} y \mathrm{~N}$. In Hertz's law the contact force is $F_{s_{H}}(y)=k_{s} y^{3 / 2}=5 \cdot 17 \times 10^{9} y^{3 / 2} \mathrm{~N}$. This means that $F_{s_{b}}>F_{s_{H}}$ for $0<y<6.6 \times 10^{-6}$ and $F_{s_{b}}<F_{s_{H}}$ for $y>6.6 \times 10^{-6}$. In our case (Figure 2) the penetration of the contact in the beam is $y<4.5 \times 10^{-6}$.

In Figure 2 it is shown that the influence of the stiffening character of Hertz's contact law is small because the high resonance peaks do not bend off much. Only in the top of the $1 / 3$ subharmonic resonance peak near $76 \mathrm{~Hz}$ a small part of the $1 / 3$ subharmonic branch is unstable, caused by the stiffening effect. The influence of the stiffening character of the contact law is low because the maximum penetration of the elastic contact in the beam does not vary very much compared to the maximum amplitude of the system, and the contact time of the beam and the elastic contact is low compared to the period of the periodic solutions.

The highest $1 / 2$ and $1 / 3$ subharmonic resonance peaks in Figure 2 are related to the harmonic resonance peak. The lower $1 / 3$ (near $37 \cdot 1 \mathrm{~Hz}$ ) and $1 / 5$ (near $62 \cdot 2 \mathrm{~Hz}$ ) subharmonic solutions are related to the second superharmonic resonance peak near $12 \cdot 4 \mathrm{~Hz}$. The $1 / 2$ (near $16 \cdot 1 \mathrm{~Hz}), 1 / 4$ (near $33 \mathrm{~Hz}$ ), $1 / 7$ (near $58 \mathrm{~Hz}$ ) and $1 / 10$ (near $83 \mathrm{~Hz}$ ) subharmonic solutions are related to the third superharmonic resonance peak near $8 \cdot 1 \mathrm{~Hz}$. The $1 / 3$ (near $18.4 \mathrm{~Hz}$ ), 1/5 (near $31 \mathrm{~Hz}$ ), 1/9 (near $56 \mathrm{~Hz}$ ) and $1 / 13$ (near $81 \mathrm{~Hz}$ ) subharmonic solutions are related to the fourth superharmonic resonance peak near $6 \cdot 0$ $\mathrm{Hz}$ (not visible in Figure 2).

On the harmonic branch and every subharmonic branch one or more periodic doubling routes leading to chaos exist. (These are not shown in Figure 2; the periodic doubling routes exist at the unstable parts of the branches marked by two flip bifurcations.) On the $1 / 2$ subharmonic branch the qualitative dynamics change very much for small frequency variations near $40 \mathrm{~Hz}$. This is caused by the fact that near $40 \mathrm{~Hz}$ grazing impact occurs: the beam just touches the elastic contact. For frequencies lower than $40 \mathrm{~Hz}$ in the $1 / 2$ subharmonic solutions the beam hits the contact twice per period, whereas for frequencies larger than $40 \mathrm{~Hz}$ in the $1 / 2$ subharmonic solutions the beam hits the contact once per period. Because the contact law is continuous, the system response does not change dramatically at grazing impact; no grazing bifurcations can be found in this system. However, as for discontinuous impacts, one would expect that the system response changes very much in the neighbourhood of grazing impact: i.e., if the penetration increases. Because of the grazing impact near $40 \mathrm{~Hz}$, the maximum displacements of the system increase and two periodic doubling routes leading to chaos were found in that frequency area. Only the $1 / 4$ subharmonic solutions of these period doubling routes are shown in Figure 2. Grazing impact also occurs at the 1/3 subharmonic resonance peak near $66 \mathrm{~Hz}$.

\section{TWO-DOF MODEL}

By using the component mode synthesis method, the system can be reduced to two DOF's. Then, in the model one residual flexibility mode and one free-interface eigenmode $\left(f_{1}=13 \cdot 1 \mathrm{~Hz}\right)$ are included. The two-DOF model has two eigenfrequencies: $f_{1}=13 \cdot 1 \mathrm{~Hz}$ and $f_{2}=125 \cdot 1 \mathrm{~Hz}$. Notice that because only one free-interface eigenmode is included, the second eigenfrequency is inaccurate. Because the second eigenfrequency of the beam, $f_{2}=117 \cdot 2 \mathrm{~Hz}$, lies close to the second eigenfrequency of the two-DOF model, still this 


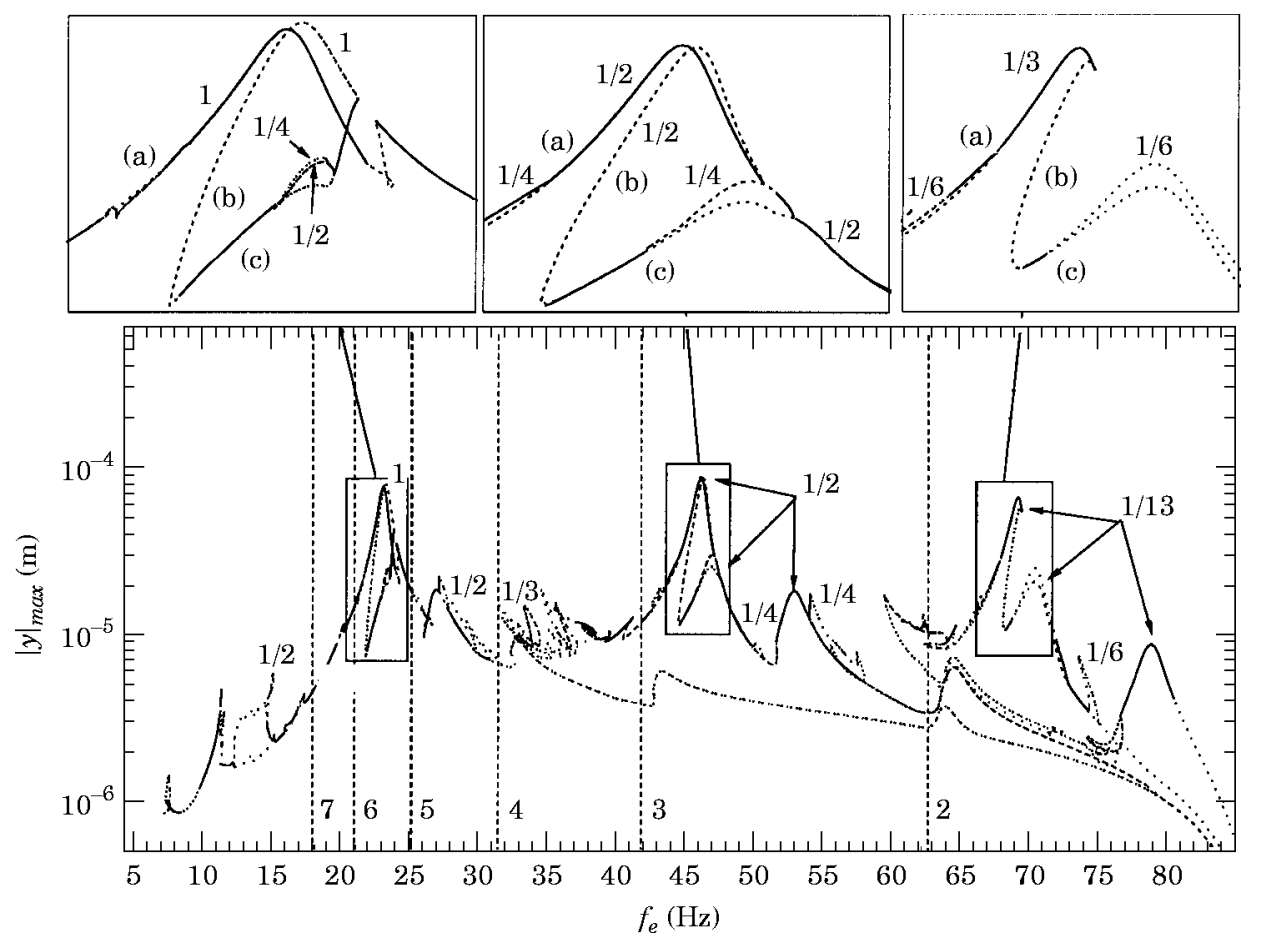

Figure 4. The maximum displacements of the periodic solutions of the 2-DOF model; $\xi_{m}=0.01 . \cdots$, Unstable; — , stable.

model is suitable to show the effect of adding one DOF to the system on the system response. In the two-DOF model, besides the linear damper in the middle, extra damping is added by means of modal damping of the two eigenmodes with modal damping coefficient $\xi_{m}=0.01$. Extra modal damping is added because it is impossible to calculate periodic solutions without modal damping due to the low damping level of the system (without modal damping all periodic solutions are numerically unstable) and in practice also modal damping is present.

In Figure 4 are shown the maximum absolute displacements $|y|_{\max }$ of the middle of the beam, occurring in the periodic solutions for varying excitation frequency of the two-DOF model. The figure shows again the harmonic, $1 / 2$ subharmonic and $1 / 3$ subharmonic solutions, now with extra resonance peaks. The extra resonance peaks on the harmonic branch are superharmonic resonance peaks of the second eigenfrequency of the model: a fifth superharmonic resonance peak (near $26 \mathrm{~Hz}$ ), a fourth superharmonic resonance peak (near $32 \mathrm{~Hz}$ ), a third superharmonic resonance peak (near $44 \mathrm{~Hz}$ ) and a second superharmonic resonance peak (near $64 \mathrm{~Hz}$ ). These superharmonic resonance peaks cause an additional resonance peak on the $1 / 2$ subharmonic and $1 / 3$ subharmonic branches. These subharmonic branches have three peaks, where the third peak is related to the fifth superharmonic resonance peak (near $26 \mathrm{~Hz}$ ) of the second eigenfrequency of the model.

The vertical dashed lines in Figure 4 show the frequency at which the superharmonic resonance peaks of the second eigenmode are expected (for $f_{2}=125 \cdot 1 \mathrm{~Hz}$ ). These dashed lines show that the fifth, fourth, third and second superharmonic resonances occur at a higher frequency than expected. The second harmonic resonance peak (not visible in 
Figure 4) occurs near $126 \cdot 5 \mathrm{~Hz}$, so the second "eigenfrequency" $\dagger$ increases from $125 \cdot 1 \mathrm{~Hz}$ to $126.5 \mathrm{~Hz}$. This does not explain the higher frequencies for which superharmonic resonances occur; the fifth superharmonic resonance peak occurs near $26.5 \mathrm{~Hz}$ and $26.5 \times 5=132.5 \mathrm{~Hz}>126.5 \mathrm{~Hz}$. In a bilinear system with $\alpha=790$, which shows a similar shift of the harmonic resonance peaks as in our system (see section 3 ), the superharmonic resonances occur at the same higher frequencies. This means that the higher superharmonic resonance frequencies are not caused by the stiffening character of the contact law of Hertz. Investigation of the periodic solutions in the superharmonic resonance peaks shows that the closer the superharmonic resonance peak lies to the first harmonic resonance peak, the greater the penetration of the elastic contact in the beam and the lower the relative time per period for which the elastic contact is "connected" to the beam. Furthermore, the superharmonic resonance peaks which lie close to the first harmonic resonance peak are not dominated only by the second "eigenfrequency" but also by the first "eigenfrequency". This means that the closer a $n$th superharmonic resonance peak lies to the first harmonic resonance peak, the more the $n$th superharmonic resonance frequency will be increased from $f_{2} / n$ to $\left(f_{1}+f_{2}\right) / n$.

Near the first harmonic resonance peak $(23 \mathrm{~Hz})$ two separated harmonic branches coexist ((a) and (b)). Both branches have a harmonic resonance peak. One branch (b) is unstable at the top. The unstable part on this branch is marked by two cyclic fold bifurcations and underneath the unstable part a stable branch (c) exists with a period doubling route to chaos. Investigation of the periodic solutions shows that in the upper (unstable) part of the branch (b) the beam hits the contact twice per period and in the lower (stable) part of the branch (c) the beam hits the contact once per period (see also the later Figure 6 in this section). Near the two cyclic fold bifurcations grazing impact occurs. At the top of the other (stable) harmonic branch (a) the beam hits the contact twice per period. Furthermore, the periodic solutions of the upper unstable part (b) are dominated by only the first "eigenfrequency" of the system, where the periodic solutions of the other branches (a) and (c) are dominated by both the first and the second "eigenfrequency". Apparently, because of the greater velocity of the beam at impact, the higher modes of the system are excited. As mentioned before, the superharmonic resonances near the first harmonic resonance peak occur at a higher frequency than expected. Because of this, the sixth superharmonic resonance peak also occurs at a higher frequency than expected. Since branch (b) is dominated by the first "eigenfrequency" only and branch (a) is dominated by both the first and the second "eigenfrequency", it can be concluded that the resonance peak on the stable branch (a) near $23 \mathrm{~Hz}$ is the sixth superharmonic resonance peak $\left(\left(f_{1}+f_{2}\right) / n=(13 \cdot 1+125 \cdot 1) / 6=23 \mathrm{~Hz}\right)$ and the resonance peak on the unstable branch (b) is the first harmonic resonance peak.

In the $1 / 2$ and $1 / 3$ subharmonic resonance peaks the beam also hits the contact twice per period, whereas in the single-DOF model the beam hits the contact once per period near the harmonic, $1 / 2$ subharmonic and $1 / 3$ subharmonic resonance peaks. In Figure 4 it is shown that near the $1 / 2$ subharmonic and $1 / 3$ subharmonic resonance peaks no separated branches coexist as they do near the harmonic resonance peak. However, near these subharmonic peaks similar behaviour is found as that near the harmonic resonance peak. With an increase of frequency from $45 \mathrm{~Hz}$ to $47 \mathrm{~Hz}$ on the $1 / 2$ subharmonic branch (a), the maximum displacement increases until the top whereafter it decreases until the branch becomes unstable via a cyclic fold bifurcation. The branch (b) turns back and simultaneously the maximum displacement increases again. Near the cyclic fold bifurcation

\footnotetext{
$\dagger$ Because the model is non-linear, one cannot speak of the eigenfrequencies of the model. Here, "eigenfrequency" is used for the frequency at which the model resonates.
} 
grazing impact occurs; however, in both the stable (a) and the unstable (b) branch the beam again hits the contact twice per period. Further decreasing of the frequency results in a decrease of amplitude of the unstable branch which then becomes stable via a cyclic fold bifurcation at $44.5 \mathrm{~Hz}$ and turns back again with increasing frequency. In this stable branch (c) the beam hits the contact once per period (near $44.5 \mathrm{~Hz}$ grazing impact occurs). Further increasing the frequency leads to a resonance peak where the branch is unstable marked by two flip bifurcations. Here a period doubling route leading to chaos exists. Only the $1 / 4$ subharmonic solutions of this period doubling route are shown in Figure 4 . At the top of the $1 / 2$ harmonic branch (c) at $47 \mathrm{~Hz}$ again grazing impact occurs, although for decreasing and increasing frequency in the $1 / 2$ subharmonic solutions the beam hits the contact once per period.

The $1 / 2$ subharmonic resonance peak near $47 \mathrm{~Hz}$ on branch (c) cannot be related directly to a superharmonic resonance peak. Investigation of the period solutions shows that again the stable $1 / 2$ subharmonic branches (a) and (c) are dominated by both the first and the second "eigenfrequencies" while the unstable branch (b) (marked by two cyclic fold bifurcations) is dominated by the first "eigenfrequency" only. Near the $1 / 3$ subharmonic resonance peak similar behaviour was found.

The subharmonic solutions related to the superharmonic resonance peaks of the first resonance peak, which dominated the response of the single-DOF model between the highest harmonic, $1 / 2$ and $1 / 3$ subharmonic peaks, could not be found in the two-DOF model. Only the $1 / 3$ subharmonic solution related to the second superharmonic resonance peak of the first eigenmode could be found near $33 \mathrm{~Hz}$ and this branch is fully unstable now. Furthermore, now more period doubling routes leading to chaos are found on the harmonic and subharmonic branches, and in the frequency ranges $30-37 \mathrm{~Hz}$ and $59-66 \mathrm{~Hz}$ chaotic behaviour was found.

Notice that, in contrast to the frequency response of the single-DOF model, the $1 / 2$ subharmonic and $1 / 3$ subharmonic resonance peaks are now lower than the harmonic resonance peak. This is caused by the anti-resonance near $85 \mathrm{~Hz}$. Furthermore, the frequencies at which the harmonic and subharmonic resonance peaks occur are decreased by $2 \mathrm{~Hz}$. The reason for this is that the beam now hits the contact twice per period in the peaks, whereas the response at the peaks is dominated by the first two "eigenfrequencies". In comparison with the linear system, the first eigenfrequency is moved from $13 \cdot 1 \mathrm{~Hz}$ to $23 \cdot 1 \mathrm{~Hz}$. The second eigenfrequency has not changed very much $(125 \cdot 1 \mathrm{~Hz} \rightarrow 126 \cdot 5 \mathrm{~Hz})$.

In Figure 5 are shown the maximum displacements occurring in the periodic solutions if the model damping coefficient is decreased to $\xi_{m}=0.001$. The response of the system is similar to the response for $\xi_{m}=0 \cdot 01$. However, now the resonance peaks are higher. Nevertheless, in the highest resonance peaks the beam still hits the contact twice per period. Furthermore, the unstable parts on the branches (most of them with period doubling routes) have become larger. Again, the subharmonic solutions caused by superharmonic resonance peaks of the first harmonic resonance peak could not be found. One additional $1 / 14$ unstable subharmonic branch was found near $57 \mathrm{~Hz}$.

The periodic solutions of the two-DOF model at $f_{e}=23.0 \mathrm{~Hz}$ are shown in Figure 6 . The figure shows that the periodic solutions of branches (a) and (b) hit the contact twice and that, in comparison to the periodic solution of the single-DOF model (Figure 3), in the periodic solutions of the two-DOF model higher frequencies are present.

\section{FOUR-DOF MODEL}

In this section the system is reduced to four DOF's. Simulations with the system reduced to five DOF's showed no major differences, so it may be concluded that the four-DOF 


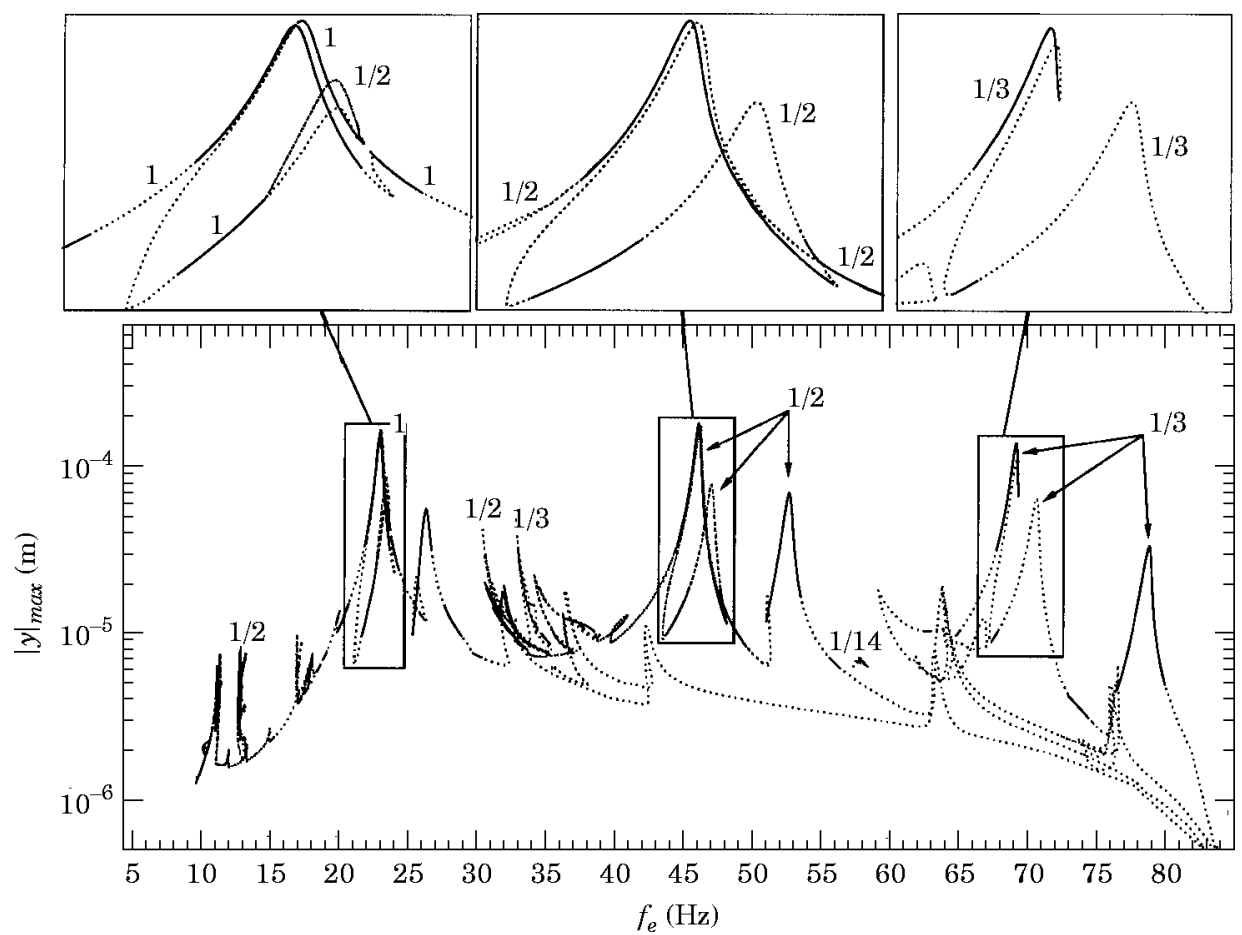

Figure 5. As Figure 4, but $\xi_{m}=0 \cdot 001$.

model is a good approximation for the unreduced system in the frequency range under investigation. In the four-DOF model three free-interface eigenmodes are included $\left(f_{1}=13 \cdot 1 \mathrm{~Hz}, f_{2}=117 \cdot 5 \mathrm{~Hz}, f_{3}=326 \cdot 5 \mathrm{~Hz}\right)$ and one residual flexibility mode. The model is approximately valid up to $500 \mathrm{~Hz}$. The eigenfrequencies of the four-DOF model are $f_{1}=13 \cdot 1 \mathrm{~Hz}, f_{2}=117 \cdot 5 \mathrm{~Hz}, f_{3}=326 \cdot 5 \mathrm{~Hz}$ and $f_{4}=749 \cdot 5 \mathrm{~Hz}$, where the last frequency is caused by the residual flexibility mode and is not accurate. Again in the four-DOF model,

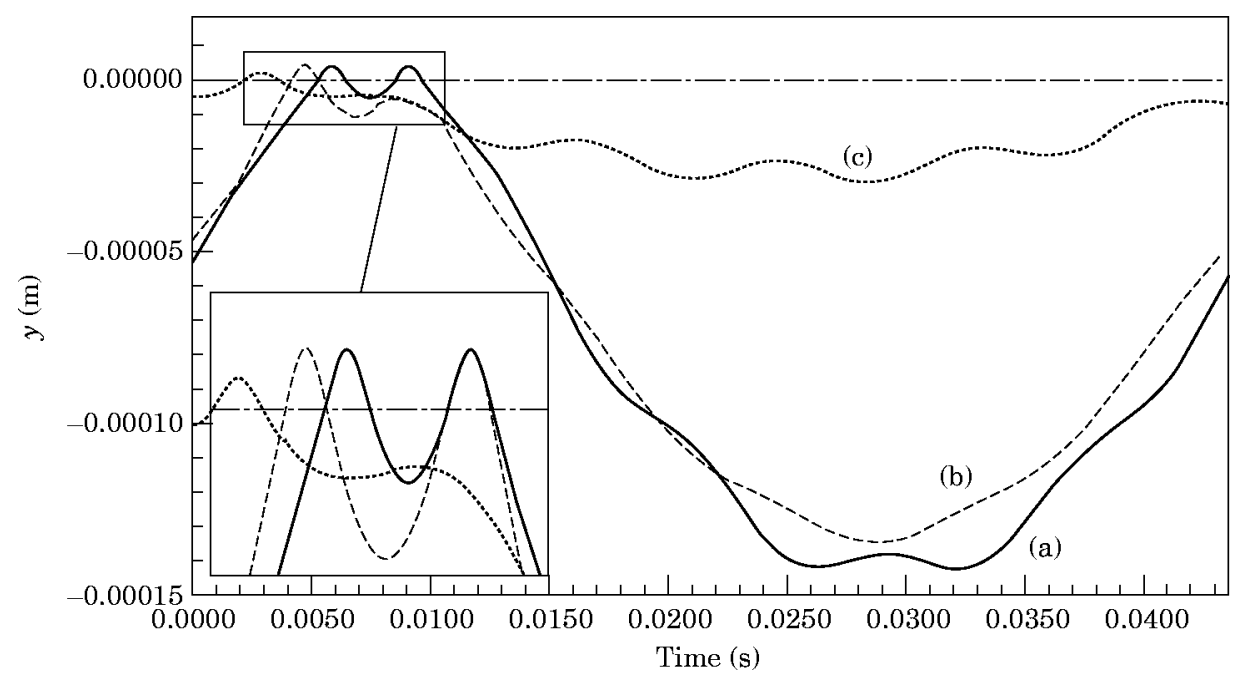

Figure 6. The periodic solutions of the 2-DOF model at $f_{e}=23 \cdot 0 \mathrm{~Hz} ; \xi_{m}=0.001$. 


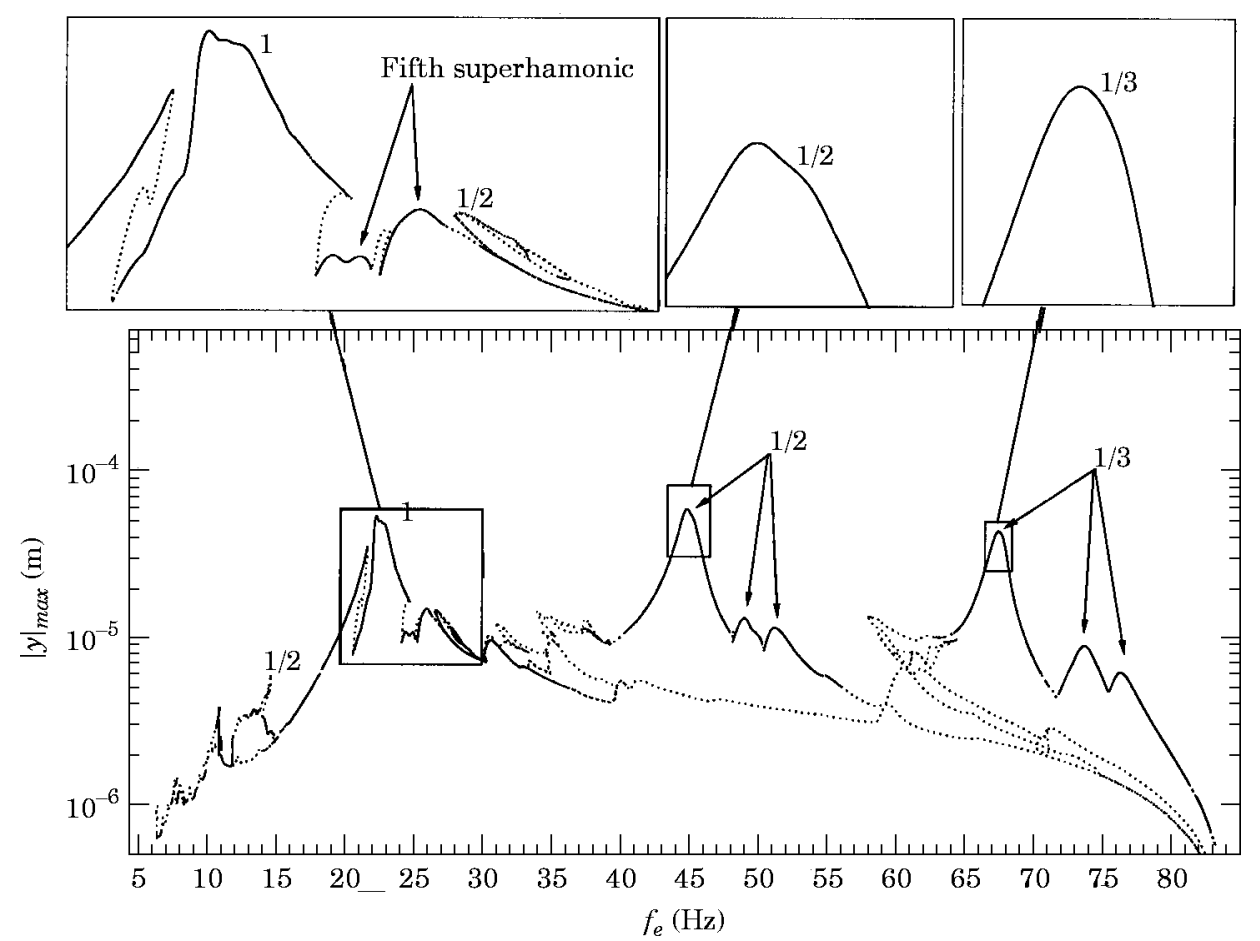

Figure 7. The maximum displacements of the 4 -DOF model; $\xi_{m}=0 \cdot 01 . \cdots$, unstable; - , stable.

besides the linear damper in the middle, extra damping is added by means of modal damping of the four eigenmodes with modal damping coefficient $\xi_{m}$.

In Figure 7 are shown the maximum absolute displacements $|y|_{\max }$ of the middle of the beam occurring in the periodic solutions for varying excitation frequency of the four-DOF model with $\xi_{m}=0 \cdot 01$. Again, the response is dominated by the harmonic resonance peak and $1 / 2$ and $1 / 3$ subharmonic resonance peaks which are related to the first harmonic resonance peak. Also again, superharmonic resonances exist at the harmonic branch which are related to the second and third "eigenfrequencies" of the system and again these superharmonic resonance peaks occur at a higher frequency than expected just as in the two-DOF model. These superharmonic resonance peaks result in additional resonance peaks on the $1 / 2$ and $1 / 3$ subharmonic solutions. The first (highest) peak on the branches with subharmonics is related to the first harmonic resonance peak. The other two peaks on the $1 / 2$ and $1 / 3$ subharmonic branches are dominated by both the second and third "eigenfrequencies". In the previous section we saw that every resonance peak near the first harmonic resonance peak is projected on the $1 / 2$ and $1 / 3$ subharmonic branches. If the extra two peaks on the $1 / 2$ and $1 / 3$ subharmonic branches are projected back on the first harmonic resonance peak, these two peaks have to be caused by the fifth superharmonic resonance peak near $27 \mathrm{~Hz}$ of the second "eigenfrequency" (see the inset of Figure 7). Because of superharmonic resonance of the third "eigenfrequency", the fifth superharmonic resonance peak is divided into two peaks: one peak is high and one peak is much lower (with two peaks on it). Because the fifth superharmonic resonance peak is divided into two peaks, also two extra peaks occur on the $1 / 2$ and $1 / 3$ subharmonic branches.

In contrast to the two-DOF model, in the four-DOF model no second harmonic branch was found near the first harmonic resonance peak. Still, there might be a second harmonic 
branch but the $1 / 2$ and $1 / 3$ subharmonic resonance peaks do not indicate that one exists. Also, because in the four-DOF model the second eigenfrequency is moved from $125.1 \mathrm{~Hz}$ to $117.5 \mathrm{~Hz}$, in comparison with the two-DOF model, now the frequency for which the sixth superharmonic resonance peak occurs has also decreased. In the two-DOF model the sixth superharmonic resonance peak was responsible for the existence of the second harmonic branch. The harmonic, $1 / 2$ and $1 / 3$ subharmonic resonance peaks are stable and the beam hits the contact twice per period. In the two extra peaks on the $1 / 2$ and $1 / 3$ subharmonic solutions the beam hits the contact once per period.

In Figure 7 it is shown that fewer period doubling routes on the harmonic, $1 / 2$ and $1 / 3$ subharmonic branches exist than in the two-DOF model with $\xi_{m}=0 \cdot 01$. This is caused by the fact that because now four eigenmodes are damped with modal damping coefficient $\xi_{m}=0 \cdot 01$, and hence the amount of damping in the system is increased which results in less non-linear phenomena. Nevertheless, in the frequency areas $30-38 \mathrm{~Hz}$ and $58-63 \mathrm{~Hz}$ chaotic behaviour was found.

The response of the system if the modal damping coefficient is decreased to $\xi_{m}=0.001$ is shown in Figure 8. Compared to Figure 7, now all sub- and superharmonic resonance peaks increase and in the frequency domain in which the harmonic resonance peak exists many superharmonic resonance peaks can be seen. These superharmonic resonance peaks are not related to one "eigenfrequency" of the model. The superharmonic resonance peaks in the frequency domain $24-26 \mathrm{~Hz}$ are again dominated by the second and third "eigenfrequency" of the model, and they are responsible for the two extra resonance peaks on the $1 / 2$ and $1 / 3$ subharmonic branches. Again in these peaks the beam hits the contact once per period. The higher peaks in the frequency domain $22-23 \mathrm{~Hz}$ are dominated by the first and second "eigenfrequencies" of the model. Some peaks are unstable and here

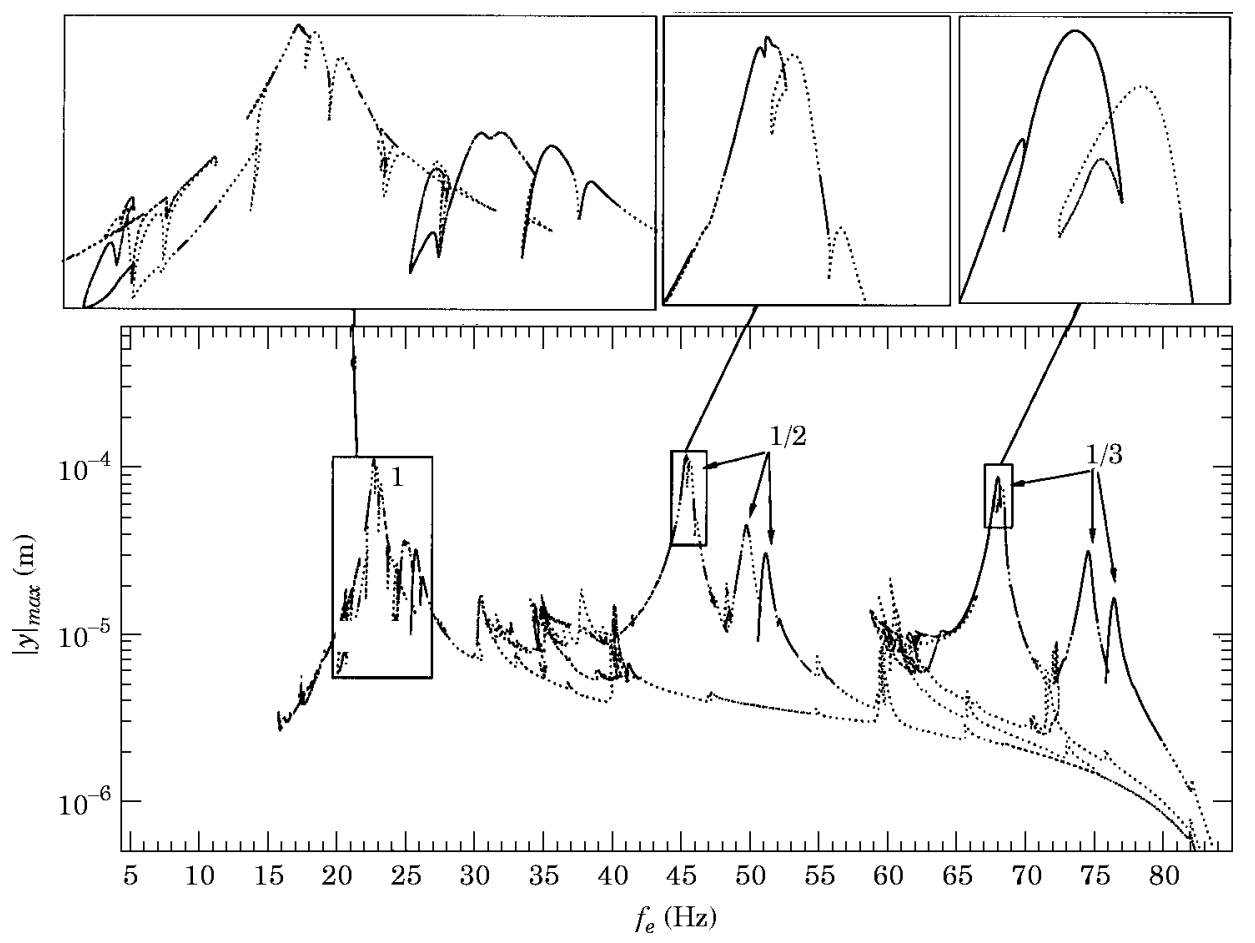

Figure 8. As Figure 7, but $\xi_{m}=0 \cdot 001$. 


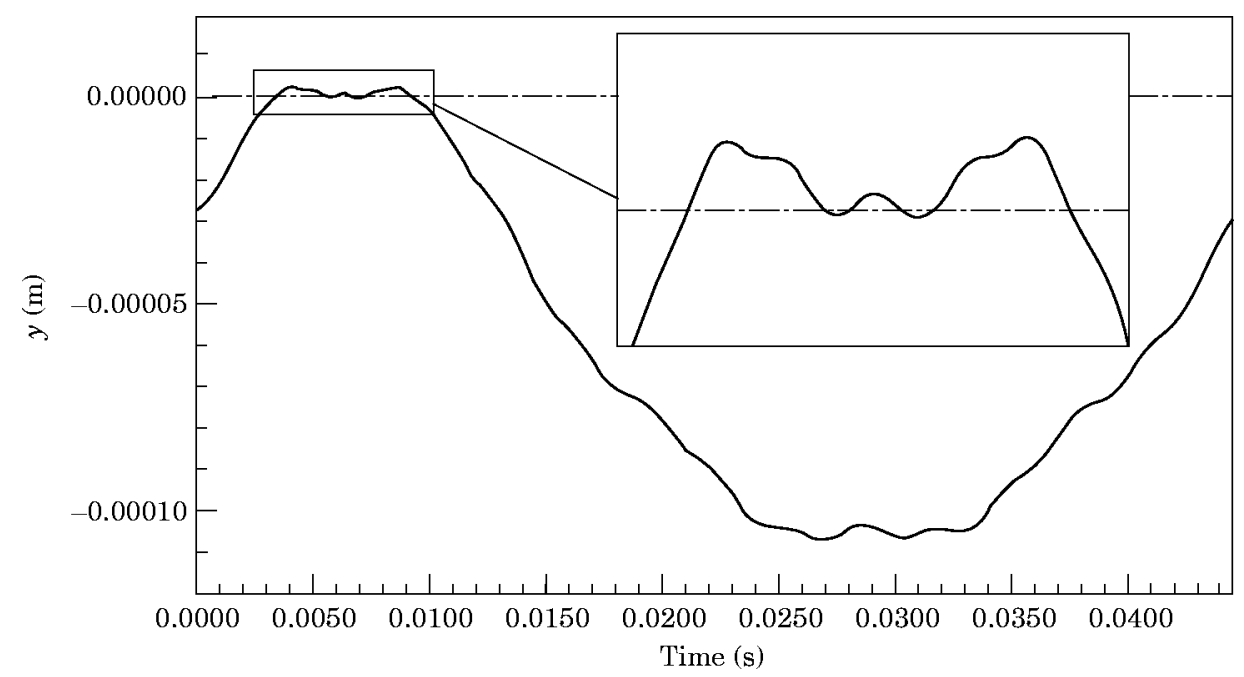

Figure 9. The periodic solution of the 4-DOF model at $f_{e}=22.5 \mathrm{~Hz} ; \xi_{m}=0.001$.

period doubling routes leading to chaos exist. In the stable peaks the beam hits the contact once per period, in the unstable peaks the beam hits the contact three times per period (see Figure 9). Also on other parts of the branches additional period doubling routes leading to chaos exist than for $\xi_{m}=0 \cdot 01$.

\section{CONCLUSIONS}

Periodic solutions have been calculated for a multi-DOF beam system with an elastic stop at its middle for varying excitation frequency. The linear beam was modelled by using finite elements and reduced by using component mode synthesis. The elastic contact as modelled by using the contact law of Hertz and the periodic solutions were calculated by solving two-point boundary value problems by the multiple shooting method. By using this method it is possible to calculate stable and (very) unstable periodic solutions and in combination with a path-following method very complex branches of periodic solutions can be calculated.

The results show that the behaviour of the single-DOF system can be globally predicted in advance if the frequency for which the harmonic resonance peak occurs is known. All the superharmonic and subharmonic resonance peaks can be predicted by using the harmonic resonance frequency. However, because on every harmonic and subharmonic branch period doubling routes leading to chaos exist, the behaviour of the single-DOF system is dominated by chaotic behaviour between the highest harmonic, $1 / 2$ and 1/3 subharmonic resonance peaks.

The two-DOF model shows globally the same maximum amplitudes as the single-DOF system. However, because of the second eigenmode an additional peak is found on the $1 / 2$ and $1 / 3$ subharmonic branches. In contrast to the single-DOF model, where the beam hits the elastic contact once per period in the highest resonance peaks, now the beam hits the elastic contact twice per period in these peaks. This means that the periodic solutions near the resonance peaks are very different from those of the single-DOF model. Between the highest harmonic, $1 / 2$ and $1 / 3$ subharmonic resonance peaks the behaviour is again dominated by chaotic behaviour, which is now caused by additional period doubling routes on the harmonic, $1 / 2$ and $1 / 3$ subharmonic resonance branches. The 
subharmonics caused by superharmonic resonances of the first "eigenfrequency", which dominated the response of the single-DOF system, have disappeared.

The response of the four-DOF model shows that although the third linear eigenfrequency of the beam is four times the highest excitation frequency under investigation, this "eigenfrequency" still has a large influence on the system behaviour. The results show that the amount of damping in the model has a large influence on the frequency responses. Increasing the modal damping leads to fewer period doubling routes and smaller frequency intervals with chaotic behaviour. However, also at higher damping levels $1 / 2$ and $1 / 3$ subharmonics dominate the frequency response and the influence of the third linear eigenfrequency is still large.

Because the impact contact has been modelled using a continuous contact law, no grazing bifurcations were found. In the neighbourhood of grazing impact, i.e., the beam just touches the elastic contact, in many cases period doubling routes occur. However, this is not always the case, and not every period doubling route is in the neighbourhood of a frequency for which grazing impact occurs.

\section{ACKNOWLEDGMENT}

This work was supported by the Centre for Mechanical Engineering of the TNO Building and Construction Research, Delft, The Netherlands.

\section{REFERENCES}

1. A. B. Nordmark 1991 Journal of Sound and Vibration 145, 279-297. Non-periodic motion caused by grazing incidence in an impact oscillator.

2. H. E. Nusse, E. Otт and J. A. Yorke 1994 Physical Review E 49, 1073-1076. Border-collision bifurcations: An explanation for observed bifurcation phenomena.

3. W. Chin, E. Ott, H. E. Husse and C. Grebogi 1994 Physical Review E 50, 4427-4444. Grazing bifurcations in impact oscillators.

4. H. Hertz 1895 Gesammelte Werke, Vol. 1: Schriften vermischten Inhalts. Leipzig, Germany.

5. W. Goldsmith 1960 Impact: the Theory and Physical Behaviour of Colliding Solids. London: Edward Arnold.

6. H. M. Lankarani and P. E. Nikravesh 1994 Nonlinear Dynamics 5, 193-207. Continuous contact force models for impact analysis in multibody systems.

7. S. Fonle and S. R. Bishop 1994 Nonlinear Dynamics 6, 285-299. Bifurcations in impact oscillations.

8. R. H. B. FEY 1992 Ph.D. Thesis, Eindhoven Univeristy of Technology, Eindhoven, The Netherlands. Steady-state behaviour of reduced dynamic systems with local nonlinearities.

9. R. H. B. Fey, E. L. B. van de Vorst, D. H. van Campen, A. de Kraker, G. J. Meijer and F. H. AssincK 1994 in Nonlinearity and Chaos in Engineering Dynamics (J. M. T. Thompson and S. R. Bishop, editors), 125-139. Proceedings of the IUTAM Symposium, London, 19-23 July 1993. Chichester: John Wiley. Chaos and bifurcations in a multi-dof beam system with nonlinear support.

10. E. L. B. van de Vorst, F. H. Assinck, A. de Kraker, R. H. B. Fey and D. H. van Campen 1996 Experimental Mechanics (accepted for publication). Experimental verification of the steady-state behaviour of a beam system with discontinuous support.

11. R. R. Craig JR. 1985 in Combined Experimental/Analytical Modeling of Dynamic Structural Systems Using Substructure Synthesis (D. R. Martinez and A. K. Miller, editors), 1-31. A review of time-domain and frequency-domain component mode synthesis methods.

12. U. M. Ascher, R. M. M. Mattheis and R. D. Russell 1988 Numerical Solution of Boundary Value Problems for Ordinary Differential Equations. Englewood Cliffs, New Jersey: Prentice-Hall.

13. DIANA User's Manual 1996. DIANA, TNO Building and Construction Research, Delft, The Netherlands, 6.0 edition, 1996.

14. S. W. Shaw and P. J. Holmes 1983 Journal of Sound and Vibration 90, 129-155. A periodically forced piecewise linear oscillator. 